\title{
Eficiencia de las entidades prestadoras de salud (EPS) en Colombia por medio de análisis envolvente de datos
}

\author{
Efficiency of entities providers of health (EPH) in Colombia through \\ data envelopment analysis \\ Tomás José Fontalvo Herrera ${ }^{1}$
}

Recibido 22 de septiembre de 2015, aceptado 21 de noviembre de 2016

Received: September 22, $2015 \quad$ Accepted: November 21, 2016

\begin{abstract}
RESUMEN
En esta investigación se analiza la eficiencia técnica, puramente técnica, global, de escala y mezcla de las EPS que hacen parte del régimen Contributivo en Colombia durante el año 2011. Se utilizó la metodología no paramétrica Data Envelopment Analysis (DEA), específicamente los modelos CCR-O, BCC-O, SBM, -O-C enfocado a salidas para evaluar la eficiencia del sector, como fuente de información utilizada se tomó la Superintendencia de Salud. De las 17 entidades estudiadas, 12 entidades mostraron ser eficientes globalmente, estas fueron: Alianza Salud, Comfenalco Valle, Compensar, S.O.S, E.P.M, Comfenalco Antioquia, Sura, Famisanar, Fondo Pasivo Ferrocarriles, Golden Group, Nueva EPS y Colpatria. Las eficiencias SBM-O-C, CCR-O, BCC-O promedios del sector fueron 88,98\%, 90,91\%, $93,07 \%$, respectivamente. Con lo que se puede concluir que el sector presenta un buen desempeño en su eficiencia.
\end{abstract}

Palabras clave: Eficiencia, administración de servicios de salud, análisis envolvente de datos.

\begin{abstract}
This research examines the technical, purely technical, global, scale and mix efficiencies of the EPSs that are part of the Contributory scheme in Colombia during 2011. Nonparametric Data Envelopment Analysis (DEA) method was used, specifically the models CCR-O, BCC-O, SBM, -O-C which are focused on outputs to assess the efficiency of the sector, as a source of information the Superintendency of Health was used. From the 17 companies studied, 12 of these proved to be globally efficient, these were: Alianza salud, Comfenalco Valle, Compensar, S.O.S, E.P.M, Comfenalco Antioquia, Sura, Famisanar, Fondo Pasivo Ferrocarriles, Golden Group, Nueva EPS y Colpatria. The SBM-O-C, CCR-O, BCC-O averages efficiencies of the industry were $88.98 \%, 90.91 \%, 93.07 \%$, respectively. Thus, it can be concluded that the sector presents a good performance on its efficiency.
\end{abstract}

Keywords: Efficiency, companies providers of health services, data envelopment analysis, health services administration.

1 Universidad de Cartagena. Av. El consulado, Calle 30 No 48-152. Cartagena 1382. Cartagena de Indias, Colombia. E-mail: tfontalvoh@unicartagena.edu.co 


\section{INTRODUCCIÓN}

La prestación de servicios de salud a nivel mundial, es uno de los servicios públicos en donde se debe garantizar la pertinencia, racionalidad y la oportunidad en los contextos donde se preste. Para esto es importante el uso racional de los recursos que los usuarios y el estado asignan para la prestación de los servicios de salud. En este sentido es necesario desarrollar estudios que permitan analizar qué tan eficiente se vienen administrando los recursos que el estado provee mediante el Fondo de Solidaridad y Garantía FOSYGA a los usuarios por medio de la Unidad de Pago por Capitación UPC. De igual forma es determinante analizar qué tan eficiente es el uso de estos recursos asignados a las EPS del régimen Contributivo, específicamente cuando se revisan la operaciones internas por medio de los ingresos operacionales generados en el período y reflejados en los estados de resultados; los que están directamente relacionados y generan causalidad con la eficiencia asociada a las operaciones en la prestación de los servicios, específicamente las Empresas Prestadoras de Salud EPS, del régimen Contributivo en Colombia.

De igual manera con esta investigación se aporta a la comunidad científica criterios para evaluar a los sectores del sector salud en cualquier contexto, analizando el desempeño en términos de sus tipos de eficiencias, lo que se constituirá en un referente para evaluar a otro grupo o sector de salud, como lo plantean autores [1] al afirmar que los resultados de este tipo de investigaciones contribuyen a establecer un sistema de información para los usuarios que les permite tomar la mejor decisión en el proceso de elección de su EPS.

Para garantizar lo anterior, este trabajo de investigación le dio respuesta a las siguientes preguntas problemas.

¿Cómo calcular las eficiencias técnicas, puramente técnica, global, de escala y de mezcla para las EPS, del régimen Contributivo en Colombia? ¿Cómo son las eficiencias promedio técnica, puramente técnica, global, de escala y de mezcla para las EPS del régimen Contributivo de Colombia?

¿Cuáles son las proyecciones requeridas en las variables de salida, para alcanzar la eficiencia global en las EPS objeto de esta investigación?

\section{ANÁLISIS TEÓRICO}

El Estado colombiano a partir del año 1991, crea el Sistema Obligatorio de Garantía de Calidad, en este establece una serie de estructuras y entidades de salud para garantizar la cobertura de toda la población en Colombia, desde esta perspectiva establece diferentes tipos de entidades como son: las empresas administradoras de planes de beneficios EAPB, que dentro del régimen Contributivo se conocen como EPS, las que son el objeto de esta investigación, asimismo crea las administradoras del régimen subsidiado ARS, hoy EPS del régimen subsidiado. Y las administradoras de regímenes especiales EAS. Todas conforman la estructura para garantizar la prestación de los servicios de los usuarios en Colombia [2].

Para garantizar la cobertura de la población, los sistemas de salud deben propender por el establecimiento de políticas públicas que contribuyan con la eficiencia, lo que requiere además trabajar conjuntamente con el ciudadano, mantener una racionalidad financiera y estimular la competencia entre entidades de salud [3]. También, otras investigaciones muestran la importancia de garantizar la accesibilidad y eficiencia en las entidades de salud [4] a los ciudadanos como resultado de la racionalidad en el uso de sus recursos.

Por otro lado, es importante señalar que el Estado colombiano ha implementado sistemas de información que establecen indicadores para monitorear el desempeño de los procesos en la prestación de servicios asistenciales. Sin embargo, no existe un sistema que permita evaluar de manera global el impacto ni la eficiencia de los servicios de salud [2] en términos específicos para las EPS del régimen Contributivo.

En concordancia con lo anterior otras investigaciones plantean la necesidad de establecer medidas de control con el fin de monitorear el desempeño y uso de los recursos asociados a la prestación de servicios de salud [5]. En concordancia con lo anterior, algunos organismos de financiación internacional promueven cambios en el sistema general de salud en Colombia, con el fin de garantizar la equidad y la eficiencia de los sistemas de salud de los países en vías de desarrollo [6] como los de Latinoamérica. 


\section{EFICIENCIA EN EL SECTOR SALUD}

Autores [7] plantean la importancia de la relación entre eficiencia, eficacia y efectividad en el sector salud, lo que posibilita la solución de múltiples problemas que existen en este sector. Autores [8] españoles analizan la eficiencia hospitalaria para la comunidad autónoma en el sistema nacional de salud español, con el fin de tomar decisiones que contribuyan con la optimización de los recursos en el sector salud. Otros estudios han trabajado en la aplicación de diversos modelos de DEA en el sector salud y establecen diferentes indicadores que permiten evaluar los costos esperados confrontando estos con los resultados alcanzados [9].

Otras investigaciones [10] han estudiado recientemente como inciden las diferentes estructuras y rubros financieros en la eficiencia de las entidades de salud en Colombia. Demostrando que los resultados alcanzados por este tipo de entidades, todavía ofrecen muchas oportunidades de mejora, en el manejo de la racionalidad y uso de los diferentes recursos.

Otros estudios [11] destacan la importancia de evaluar la eficiencia técnica global en el sector salud utilizando criterios de evaluación económicas, que permitan tomar decisiones estratégicas, que contribuyan con la competitividad de las entidades de salud. Lo anterior es coherente con estudios similares desarrollados por otros autores [12] que plantean la necesidad de establecer indicadores de eficiencia que contribuyan con la atención primaria y la gestión hospitalaria, lo que es similar a lo que se analizó en esta investigación. Asimismo investigaciones en Portugal muestran la importancia de desarrollar procesos de sinergias entre entidades de salud y optimización de costos para mejorar los niveles de eficiencia en el sector salud del país [13].

\section{MODELOS DEA CCR-O, BCC-O, SBM-O-C}

Autores que han trabajado en la aplicación de modelos de eficiencia en el sector salud en Colombia, plantean la importancia [10], del modelo CCR-O que permite evaluar un conjunto de variables de entrada y analizar como inciden estas para optimizar las variables de salida de las DMU analizadas. Además [14] otras investigaciones muestran la pertinencia del modelo DEA para evaluar centros de atención primaria en México. Otros estudios destacan y aplican el modelo DEA BBC-O enfocados en salidas y plantean [15], el modelo que se muestra a continuación, el que permite calcular la eficiencia técnica pura o administrativa la cual permite evaluar las DMU objeto de la investigación de manera puntual y no radial como la eficiencia CCR-O. Investigaciones sobre el modelo BCC señalan el cuidado que hay que tener en la selección de las variables con el fin de no generar restricciones en su aplicación [16]. Asimismo otros investigadores destacan la importancia de la eficiencia global para evaluar entidades públicas [17].

Sin embargo, otros estudios científicos señalan la importancia de evaluar simultáneamente la eficiencia técnica global, la eficiencia puramente técnica y la eficiencia global [18]. Esto autores analizan los modelos de eficiencia [19] y aplican los modelos CCR-O, BCC-O Y SBM-O para analizar los diferentes tipos de eficiencia en un grupo de entidades del sector público. A continuación se presentan los modelos matemáticos previamente señalados [20]:

\section{El modelo CCR-O}

Viene dado por la siguiente expresión matemática:

$$
\begin{aligned}
& \operatorname{Max} \quad \eta \\
& \mathrm{x}_{\mathrm{o}}-X \mu \geq 0 \\
& \eta \mathrm{y}_{\mathrm{o}}-Y \mu \leq 0 \\
& \mu \geq 0
\end{aligned}
$$

\section{El modelo BCC-O}

Y el modelo BCC viene dado por la siguiente expresión matemática;

$$
\begin{aligned}
& \operatorname{Max} \eta_{B} \\
& \mathrm{x}_{\mathrm{o}}-X \lambda \geq 0 \\
& \eta_{B} \mathrm{y}_{\mathrm{o}}-Y \lambda \leq 0 \\
& e \lambda=1 \\
& \lambda \geq 0
\end{aligned}
$$

\section{El modelo SBM-O}

El modelo SBM-O orientado a salidas se expresa mediante la siguiente expresión matemática: 


$$
\begin{gathered}
(S B M-O) \quad \rho_{o}^{*}=\operatorname{Min}_{\lambda, s^{+}} \frac{1}{1+\frac{1}{s} \sum_{r=1}^{s} \frac{s_{r}^{+}}{y_{r o}}} \\
x_{o}=X \lambda+\mathrm{s}^{-} \\
y_{o}=Y \lambda-s^{+} \\
\lambda \geq 0, \quad s^{+} \geq 0 \quad \mathrm{~s}^{-} \geq 0
\end{gathered}
$$

\section{La eficiencia de escala se define por:}

$$
\text { Eficiencia de escala }=\frac{\text { La eficiencia } C C R}{\text { Eficiencia BCC }}
$$

\section{La eficiencia de mezcla se define por:}

De esta forma tenemos una descomposición de la eficiencia no radial (SBM - O) en eficiencia radial (CCR) y eficiencia de mezcla (MIX):

$$
\text { Eficiencia de Mezcla }=\frac{\text { Eficiencia } S B N}{\text { Eficiencia } C C R}
$$

\section{METODOLOGÍA}

Como concepción epistemológica en esta investigación se tuvieron en cuenta los siguientes criterios; como concepción de verdad se tuvo un enfoque mixto; es decir, se trabajó con un enfoque racionalista y empírico, porqué la investigación se desarrolló desde una perspectiva empírica, al desarrollar procesos de verificación, observación, inferencia racional sobre los resultados alcanzados por las EPS objeto de estudio. Asimismo se consideró un universo, población y muestra, a la que se calcularon una serie de parámetros para evaluar los diferentes tipos de eficiencia. También se desarrolló un análisis racional para definir las variables y analizar, comprender e interpretar los diferentes resultados asociados a los modelos CCR-O, BCC-O, SBM-O-C.

Para el desarrollo de esta investigación como lógica del método se tuvo un enfoque racionalista, con lo que se pudo deducir y proponer las variables asociados las EPS objeto de la investigación. También se consideró un enfoque empírico, teniendo en cuenta que para el proceso de medición de la eficiencia se trabajó con un enfoque empírico, toda vez que se consideraron rubros asociados a la experiencia de las EPS objeto de la investigación. Ello fue desarrollado, por medio de algunos pasos que a la postre permitieron calcular los diferentes tipos de eficiencias.

La esencia de la ciencia estuvo relacionado con el objeto de la investigación, como fueron los datos empíricos asociados con los indicadores de eficiencia calculada, pero fue determinante el sujeto investigador, teniendo en cuenta que mediante los procesos de análisis y racionalidad se pudo definir, analizar, interpretar y comprender el fenómeno objeto de esta investigación. Por tanto el origen del conocimiento científico generado surgió de la experiencia y de la razón del investigador.

La concepción de la realidad se consideró fraccionada y sistémica a la vez, teniendo en cuenta que se analizaron y estudiaron las diferentes EPS desde su individualidad, pero además se consideró la totalidad, para identificar las EPS eficientes que se constituyeron en DMU de referencia, para las EPS ineficientes. El estado de la realidad se entendió dialectico, toda vez que se estudiaron las EPS eficientes y no eficientes, y de estas diferencias se pudieron establecer las EPS referentes para evaluar la totalidad de EPS de este trabajo de investigación.

También se consideraron a las organizaciones objeto de esta investigación organicista y holista, pues se consideraron como un todo para poder calcular las eficiencias promedios, relacionados con los tres métodos utilizados para el modelo propuesto como son el CCR-O, BCC-O y SBM$\mathrm{O}-\mathrm{C}$. También se entendieron los grupos de DMU o EPS seleccionadas, como entes sociales que deben analizar y comprender sus niveles de eficiencia, para que esto repercuta en los contextos sociales donde cada una de estas interviene.

Esta investigación muestra los resultados de la investigación realizada en las EPS de Colombia en el año 2011 mediante la metodología Data Envelopment Analysis (DEA), con los modelos CCR-O, BCC-O y SBM,-O-C enfocados en salidas.

En este estudio se utilizó un análisis de la eficiencia técnica, puramente técnica y global en las EPS que hacen parte del régimen Contributivo en Colombia durante el 2011, con lo que se pudo calcular las eficiencias de mezcla y de escala para ello fue necesario definir las variables de entradas y salidas. 
Con lo anterior se determinaron las proyecciones requeridas de las variables de salida de los ingresos, ingresos del fondo de solidaridad y garantía FOSYGA y los Ingresos Operacionales que se requieren en el período de estudio.

Para el estudio de eficiencia se tuvo en cuenta las 17 EPS en Colombia. En la Tabla 1 se presentan dichas instituciones.

Tabla 1. EPS del régimen Contributivo consideradas.

\begin{tabular}{|l|}
\hline DMU's \\
\hline Aliansalud \\
\hline Comfenalco Valle \\
\hline Compensar \\
\hline Coomeva \\
\hline EPM \\
\hline Comfenalco Antioquia \\
\hline Sura \\
\hline Famisanar \\
\hline Fondo de Pasivo Ferrocarriles \\
\hline Golden Group \\
\hline Nueva EPS \\
\hline Colpatria \\
\hline Salud Total \\
\hline Saludvida \\
\hline Sanitas \\
\hline S.O.S. \\
\hline Solsalud \\
\hline
\end{tabular}

Fuente: Producción del autor.

La información con la que se realizó la investigación fue recolectada de la Superintendencia de Salud, año 2011, Para esto fue necesario identificar y seleccionar las variables que se ajustaran a los tres modelos de evaluación de eficiencia DEA utilizados.

La Tabla 2 muestra los valores (magnitud) y las variables de entrada y salida para cada una de las EPS que se consideraron en el estudio. Para el cálculo de las eficiencias se utilizaron los modelos DEA CCR-O, BCC-OY SBM-O C y se utilizó el software DEA Solver PRO 09, con el que se pudo realizar el análisis de los diferentes niveles de eficiencia de las EPS del régimen Contributivo en Colombia. A continuación se muestran las variables de entrada y salida que se consideraron para la aplicación de los modelos DEA utilizados en esta investigación.

\section{Variables de entrada}

Activo total: Representa la suma del activo corriente y del activo no corriente.

Inversiones: Corresponde a los rubros de inversiones en acciones, cuotas o partes de interés social, títulos valores, papeles comerciales o cualquier otro documento negociable adquirido por la empresa con carácter temporal o permanente.

Cuentas por cobrar FOSYGA: Son las cuentas a cobrar al Estado colombiano, diferentes al plan obligatorio de salud asociado a los derechos que posee una entidad de salud sobre terceras personas naturales y/o jurídicas pendientes de cobro a una determinada fecha.

Activo fijo: Son los activos relacionados con propiedad planta y equipo, con los que cuentan las EPS.

\section{Variables de salida}

Ingresos UPC: Unidad de pago por captación de usuarios.

Ingresos recobro del FOSYGA: Ingresos resultado de presentar cuentas al Estado colombiano por concepto de tecnologías de salud, no incluidas en el plan obligatorio de salud autorizadas por el comité técnico científico u ordenadas por fallos de tutelas.

Investigaciones consideran importante estos recursos del FOSYGA para la prestación del servicios de salud cuando se analizan la estructura de las instituciones prestadoras de servicios de salud en Colombia [21].

Ingreso operacional: Ingresos producto de la actividad económica principal de la empresa.

\section{RESULTADOS}

Con el propósito de realizar un análisis racional de los resultados producto del cálculo de los diferentes niveles de eficacia a continuación se analizan la correlación de las diferentes variables establecidas, posteriormente se calculan y analizan los diferentes tipos de eficiencias como son la técnica, la puramente técnica, la global, de 
Tabla 2. Magnitud de las variables de entradas y salidas de entidades que hacen parte del régimen contributivo en Colombia.

\begin{tabular}{|c|c|c|c|c|c|c|c|}
\hline EPS & $\begin{array}{l}\text { (I) Activo } \\
\text { total }\end{array}$ & $\begin{array}{c}\text { (I) } \\
\text { Inversiones }\end{array}$ & $\begin{array}{c}\text { (I) } \\
\text { Total } \\
\text { cuentas } \\
\text { por cobrar } \\
\text { FOSYGA }\end{array}$ & $\begin{array}{c}\text { (I) } \\
\text { Activo fijo } \\
\text { propiedad } \\
\text { planta y } \\
\text { equipo }\end{array}$ & $\begin{array}{c}(0) \\
\text { Ingresos } \\
\text { UPC }\end{array}$ & $\begin{array}{c}(0) \\
\text { Ingresos } \\
\text { recobro al } \\
\text { FOSYGA }\end{array}$ & $\begin{array}{c}(0) \\
\text { Ingresos } \\
\text { operacinoales }\end{array}$ \\
\hline Aliansalud & 74.659 .289 & 19.712 .425 & 43.573 .089 & 506.726 & 196.384 .549 & 81.430 .596 & 193.387.216 \\
\hline Comfenalco Valle & 73.192 .236 & 810.002 & 36.472 .629 & 15.382 .635 & 154.675 .269 & 39.994 .397 & 138.684 .343 \\
\hline Compensar & 147.183 .633 & 15.127 .388 & 75.399 .989 & 4.243 .481 & 408.946 .118 & 108.062 .571 & 425.911 .666 \\
\hline Coomeva & 552.127 .504 & 12.757 .631 & 403.192 .739 & 12.549 .111 & 1.412 .872 .026 & 384.858 .018 & 1.464 .245 .760 \\
\hline EPM & 11.497 .792 & 8.997 .307 & 0 & 0 & 11.851.157 & 0 & 8.712 .201 \\
\hline Comfenalco Antioquia & 55.003 .905 & 4.500 .000 & 43.468 .103 & 1.489 .166 & 177.047 .201 & 50.863 .177 & 177.934 .783 \\
\hline Sura & 247.430 .381 & 78.407 .570 & 101.337 .602 & 17.131.377 & 696.932 .272 & 198.384 .339 & 713.300 .668 \\
\hline Famisanar & 254.676 .457 & 21.000 & 176.767 .704 & 8.508 .024 & 669.227 .215 & 124.616 .077 & 666.839 .628 \\
\hline $\begin{array}{l}\text { Fondo De Pasivo } \\
\text { Ferrocarriles }\end{array}$ & 39.370 .561 & 16.297 .183 & 3.739 .882 & 229.932 & 54.289 .171 & 0 & 104.784 .504 \\
\hline Golden Group & 8.873 .885 & 0 & 1.003 .682 & 686.940 & 24.376 .955 & 522.175 & 25.567 .417 \\
\hline Nueva Eps & 1.058 .955 .212 & 3.191 .139 & 725.870 .151 & 2.942 .152 & 1.903.254.182 & 788.690 .698 & 1.811.061.005 \\
\hline Colpatria & 29.626 .063 & 18.584 .149 & 982.376 & 6.142 & 44524895 & 6.509 .171 & 43.522 .988 \\
\hline Salud Total & 365.396 .570 & 198.238.511 & 75.315 .458 & 35.242 .132 & 787.461 .401 & 109.833 .981 & 817.409 .281 \\
\hline Saludvida & 68.974 .504 & 4.785 .992 & 7.520 .665 & 7.904 .296 & 42.480 .528 & 6.890 .123 & 29.513 .895 \\
\hline Sanitas & 379.950 .832 & 25.376 .938 & 242.987 .341 & 3.000 .112 & 589.479 .187 & 227.092 .530 & 582.135 .254 \\
\hline S.O.S. & 118.171 .938 & 1.319 .225 & 86.320 .782 & 3.688 .966 & 376.769 .004 & 89.411 .705 & 359.358 .087 \\
\hline Solsalud & 10.686 .9758 & 2.613 .716 & 46.573 .124 & 3.457 .365 & 87.208 .246 & 17.644 .963 & 87.656 .995 \\
\hline
\end{tabular}

Fuente: Información tomada de la Superintendencia de Salud 2011.

escala y de mezcla. Con los que se analizó el desempeño de las DMU objeto de investigación en sus niveles, administrativos, operativos y global.

De igual manera se estudian las eficiencias promedios del grupo de EPS de Colombia, a nivel de eficiencia técnica, puramente técnica y global. Seguidamente se analiza e interpreta los resultados asociados con las EPS que se constituyeron en referentes de eficiencia para evaluar las EPS del régimen Contributivo no eficientes de Colombia. Finalmente, se realizan y comprenden las proyecciones requeridas en las variables de salida para alcanzar la eficiencia global, por parte de las EPS clasificadas como ineficientes.

De la Tabla 3 se puede observar que existe una correlación sólida entre las variables de entrada, activo total y total cuentas por cobrar del FOSYGA con las variables de salida, ingresos UPC, Ingreso recobro al FOYGA y los ingresos operacionales. Sin embargo, cuando se revisa la correlación entre inversiones con las variables de salida ingresos UPC, ingreso recobro al FOYGA, y los ingresos operacionales, la correlación es baja; es decir, las inversiones en este grupo de organizaciones no afectan este tipo de ingresos.

Del análisis de correlación se puede observar que existe una correlación firme entre las variables de entrada y salida; es decir, que un incremento de las variables de entrada se ve reflejada en los rubros considerados como variables de salida. Sin embargo, cuando se revisa los rubros inversiones y activo fijo (propiedad planta y equipo), estos no guardan una correlación significativa con el total de cuentas por cobrar, los ingresos UPC, ingresos de recobro del FOSYGA y los ingresos operacionales, lo anterior obedece a que en este sector de la salud, estas variables no son directamente dependientes.

La Tabla 4 presenta los puntajes de eficiencia según el modelo CCR-O, BCC-OY SBM-O, -C enfocado 
Tabla 3. Correlación entre las variables.

\begin{tabular}{|l|c|c|c|c|c|c|c|}
\cline { 2 - 8 } \multicolumn{1}{c|}{} & $\begin{array}{c}\text { Activo } \\
\text { total }\end{array}$ & Inversiones & $\begin{array}{c}\text { Total cuentas } \\
\text { por cobrar } \\
\text { FOSYGA }\end{array}$ & $\begin{array}{c}\text { Activo fijo } \\
\text { propiedad } \\
\text { planta y equipo }\end{array}$ & $\begin{array}{c}\text { Ingresos } \\
\text { UPC }\end{array}$ & $\begin{array}{c}\text { Ingresos } \\
\text { recobro al } \\
\text { FOSYGA }\end{array}$ & $\begin{array}{c}\text { Ingresos } \\
\text { operacionales }\end{array}$ \\
\hline Activo total & 1 & & & & & & \\
\hline Inversiones & 0,1504 & 1 & & & & & \\
\hline $\begin{array}{l}\text { Total cuentas por } \\
\text { cobrar FOSYGA }\end{array}$ & 0,9716 & $-0,0750$ & 1 & & & & \\
\hline $\begin{array}{l}\text { Activo fijo propiedad } \\
\text { planta y equipo }\end{array}$ & 0,2314 & 0,8348 & 0,0395 & 1 & & & \\
\hline Ingresos UPC & 0,9676 & 0,19168 & 0,9423 & 0,3167 & 1 & & \\
\hline $\begin{array}{l}\text { Ingresos recobro al } \\
\text { FOSYGA }\end{array}$ & 0,9752 & 0,00223 & 0,9847 & 0,0841 & 0,9492 & 1 & \\
\hline $\begin{array}{l}\text { Ingresos } \\
\text { operacionales }\end{array}$ & 0,9564 & 0,2144 & 0,92787 & 0,3384 & 0,9983 & 0,9340 & 1 \\
\hline
\end{tabular}

Fuente: Calculado con la información de la Superintendencia de Salud.

Tabla 4. Puntajes de eficiencias CCR-O, BCC-O, SMB-O-C, eficacia de escala y de mezcla para las EPS de Colombia.

\begin{tabular}{|l|l|l|l|l|l|l|l|}
\hline \multicolumn{1}{|c|}{ EPS } & $\begin{array}{c}\text { Score } \\
\text { CCR-O }\end{array}$ & $\begin{array}{c}\text { Score } \\
\text { BCC-O }\end{array}$ & $\begin{array}{c}\text { Score } \\
\text { SBM -O -C }\end{array}$ & $\begin{array}{c}\text { Eficiencia } \\
\text { escala }\end{array}$ & $\begin{array}{c}\text { Eficiencia } \\
\text { mezcla }\end{array}$ & RTS & $\begin{array}{c}\text { RTS de } \\
\text { proyección } \\
\text { para las EPS }\end{array}$ \\
\hline Aliansalud & 1 & 1 & 1 & 1 & 1 & Constante & \\
\hline Comfenalco Valle & 1 & 1 & 1 & 1 & 1 & Constante & \\
\hline Compensar & 1 & 1 & 1 & 1 & 1 & Constante & \\
\hline S.O.S. & 1 & 1 & 1 & 1 & 1 & Constante & \\
\hline EPM & 1 & 1 & 1 & 1 & 1 & Constante & \\
\hline Comfenalco Antioquia & 1 & 1 & 1 & 1 & 1 & Constante & \\
\hline Sura & 1 & 1 & 1 & 1 & 1 & Constante & \\
\hline Famisanar & 1 & 1 & 1 & 1 & 1 & Constante & \\
\hline Fondo de Pasivo Ferrocarriles & 1 & 1 & 1 & 1 & 1 & Constante & \\
\hline Golden Group & 1 & 1 & 1 & 1 & 1 & Constante & \\
\hline Nueva Eps & 1 & 1 & 1 & 1 & 1 & Constante & \\
\hline Colpatria & 1 & 1 & 1 & 1 & 1 & Constante & \\
\hline Coomeva & 0,9745 & 1 & 0,92106 & 0,9745 & 0,9451 & Decreciente & \\
\hline Salud Total & 0,8481 & 1 & 0,84622 & 0,8481 & 0,9977 & Decreciente & \\
\hline Sanitas & 0,7409 & 0,7912 & 0,73787 & 0,9364 & 0,9959 & & Decreciente \\
\hline Saludvida & 0,5459 & 0,6317 & 0,27845 & 0,86417 & 0,51007 & & Constante \\
\hline Solsalud & 0,3459 & 0,4006 & 0,34367 & 0,8634 & 0,9935 & & Constante \\
\hline Fute: Cal & & & 1 & & & \\
\hline
\end{tabular}

Fuente: Calculado con la información de la Superintendencia de Salud. 
a salidas. Los resultados indican que 12 de 17 entidades que hacen parte del régimen Contributivo son eficientes, esto corresponde al 70,58\% del total de DMU's evaluadas. Estudios similares en otros contextos [22] muestran resultados similares cuando se evalúan las eficiencias puramente técnica y la eficiencia global en entidades de salud.

De la Tabla 4 también se observa que las entidades como Coomeva y Salud Total, a pesar de no tener una eficiencia de 1 , muestran un buen comportamiento en sus desempeños, cuando se revisan los diferentes tipos de Eficiencia objeto de esta investigación considerando las variables seleccionadas, Sanitas presenta un desempeño aceptable. Es claro y evidente la optimización de los recursos de estas entidades cuando se trata de generar Ingresos.

Por otro lado, las entidades Saludvida y SolsaLud, tienen oportunidades de mejoras. Posibilidades de mejora que pueden optimizarse cuando se revisen y apropien de las prácticas de las EPS que se constituyeron en referentes y que presentaron mejores desempeños. Ver Tabla 4 y Figura 1.

Un análisis del conjunto de referencia muestra que la entidades Golden Group y S.O.S. son las entidades que hace parte del régimen Contributivo que más sirve de Benchmarking para las empresas que resultaron ineficientes con tres evaluaciones cada una, es decir, seis veces, lo que corresponde al 35,29\% de las entidades ineficientes. Estos resultados son coherentes con otros estudios cuando se evaluaron un grupo de instituciones de salud en México [14].

La Figura 1 muestra las veces que cada EPS eficiente evalúa a las entidades ineficientes, y se constituye
Empresas evaluadoras referentes

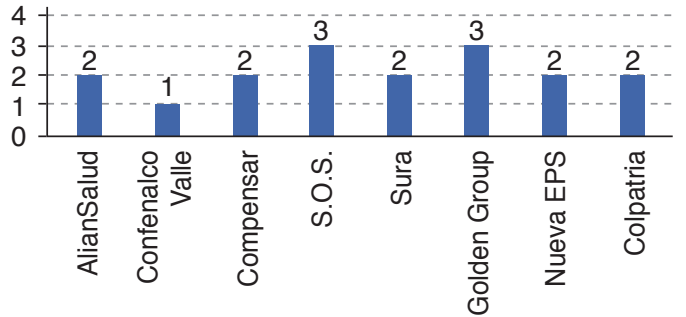

Figura 1. Número de veces que una entidad eficiente es evaluadora de las ineficientes.

en referente para mejorar los diferentes tipos de eficiencias. Lo anterior analizado desde el modelo SMB-O-C.

En la Figura 1 se observa que Golden Group y S.O.S son las EPS, que se constituyeron en las más referenciadas para evaluar al resto de EPS, con tres entidades de salud evaluadas.

El modelo DEA sugiere que para alcanzar la eficiencia, se requiere una combinación de las variables de entrada y de salidas sobre la proyección de la frontera de eficiencia. Para que una EPS sea eficiente en el modelo SBM-C-O la magnitud de aumento en la magnitud de cada variable de salida para cada entidad que hace parte del régimen Contributivo se presenta en la Tabla 5 . Se consideraron solo las variables de salida, ya que son las pertinentes para el modelo utilizado.

De la Figura 1 se observa que las EPS con mejor desempeño son las entidades S.O.S., seguida por Golden Group y por la entidad Comfenalco Antioquia, por otro lado, la entidad Solsalud presenta un desempeño bajo, seguida por Saludvida

Tabla 5. Proyección requerida en las variables de salidas para alcanzar la eficiencia.

\begin{tabular}{|l|c|c|c|c|}
\hline \multicolumn{1}{|c|}{ DMU } & Score & $\begin{array}{c}\text { Ingresos UPC } \\
\text { (Aumento) }\end{array}$ & $\begin{array}{c}\text { Ingresos recobro FOSYGA } \\
\text { (Aumento) }\end{array}$ & $\begin{array}{c}\text { Ingresos operacionales } \\
\text { (Aumento) }\end{array}$ \\
\hline Coomeva & 0,92 & 155.685 .857 & 434.538 .503 & 150.248 .685 \\
\hline Salud Total & 0,84 & 807.274 .285 & 166.949 .444 & 0 \\
\hline Sanitas & 0,73 & 804.520 .987 & 306.434 .908 & 786.798 .388 \\
\hline Solsalud & 0,34 & 255.488 .281 & 51.321 .138 .7 & 253.413 .117 \\
\hline Saludvida & 0,27 & 165.939 .912 & 0 & 173.174 .904 \\
\hline
\end{tabular}

Fuente: Calculado con la información de la Superintendencia de Salud 2011. 
y Sanitas. Coomeva, presenta una eficiencia buena de $97,45 \%$ y Salud Total obtuvo una eficiencia aceptable de $84,81 \%$.

\section{DISCUSIÓN}

Los resultados de la eficacia SBM-O muestran que 12 de 17 EPS presentan eficiencia óptima, lo que muestra que el $70,58 \%$ son eficientes; es decir, estas entidades de salud son eficientes técnicas, administrativas y globalmente, así como eficientemente en término de mezcla y de escala, estas EPS son, Alianza Salud, Comfenalco Valle, S.O.S, E.P.M, Comfenalco Antioquia, Sura Famisanar, Fondo Pasivo Ferrocarriles, Golden Group, Nueva EPS y Colpatria.

$\mathrm{Al}$ revisar la eficiencia CCR-O y confrontarlo con la eficiencia SBM-O-C estas presenta también una eficiencia de 1; es decir, el grupo de entidades de salud estudiadas muestran que no existe problemas de mezclas, relacionados con los rubros establecidos para esta investigación. Es decir, no se observan problemas de ineficiencias en las mezcla de las variables o rubros financieros que se consideraron como insumos.

Al revisar la eficiencia puramente técnica o administrativa BCC-O se observa que 14 de 17 EPS no presentan ineficiencias administrativa o en la operación. Y existen dos EPS de las 14 que presentan ineficiencia global, la que se debe a problemas en la mezcla de los insumos, a los retornos de escala y a la escala de la operación. Es decir, al escalar la operación las EPS Coomeva y Salud Total mejorarán su eficiencia.

Sanitas, Salud Vida y Sol Salud son las más ineficientes y presentan ineficiencias de retornos de escala, administrativa y globalmente, así como en la mezcla de los rubros considerados como sus insumos. También presenta ineficiencia de escala.

\section{CONCLUSIONES}

El presente estudio analiza los diferentes tipos de eficiencia como son la técnica administrativa y global, de donde se puede concluir que cuando se evalúan las EPS objeto de esta investigación, se puede aseverar que los desempeños globales se pueden definir como buenos teniendo en cuenta los resultados presentados en la Tabla 6 .
Tabla 6. Eficiencia promedio de las EPS de Colombia.

\begin{tabular}{|l|c|c|c|}
\hline $\begin{array}{c}\text { Eficiencias de las } \\
\text { EPS en Colombia }\end{array}$ & $\begin{array}{c}\text { Score } \\
\text { CCR-O }\end{array}$ & $\begin{array}{c}\text { Score } \\
\text { BCC-O }\end{array}$ & $\begin{array}{c}\text { Score } \\
\text { SBM-O-C }\end{array}$ \\
\hline Promedio & 90,91 & 93,07 & 88,98 \\
\hline
\end{tabular}

La eficiencia técnica, administrativa, global, de escala y de mezcla del grupo de EPS de las entidades que hacen parte del régimen Contributivo en Colombia cuando se considera como variable de entrada y salida muestran que las eficiencias promedio global SBM-O-C para las entidades que hacen parte del régimen Contributivo en Colombia fue de $88,98 \%$, la eficiencia técnica promedio del grupo fue $90,91 \%$ y la eficiencia administrativa promedio del grupo de EPS fue de $93,07 \%$, lo que es bueno para el régimen Contributivo. Otros estudios [23] muestran pequeñas mejoras cuando se evalúan las EPS del régimen subsidiado en Colombia.

Otras investigaciones en el sector salud muestran resultados de eficiencia que oscilan entre el 93\% y $97 \%$ de eficiencia, similares a los encontrados en esta investigación, cuando se utilizan otros criterios y técnicas para medir estas instituciones de salud [9]. Contrario a lo que plantean otras investigaciones [24], en esta investigación se pudo demostrar que las EPS del régimen Contributivo en su mayoría son eficientes, por tanto, como principal conclusión al revisar la eficacia global derivados del modelo planteado se puede afirmar que 12 de las 17 entidades que hacen parte del régimen Contributivo en Colombia, fueron eficientes para un total de $70,58 \%$, estas instituciones fueron: S Alianza Salud, Comfenalco Valle, S.O.S, E.P.M, Comfenalco Antioquia, Sura Famisanar, Fondo Pasivo Ferrocarriles, Golden Group, Nueva EPS y Colpatria.

Es importante señalar que las EPS Sanitas, Saludvida y Solsalud requieren revisar las decisiones y gestiones asociadas con las variables de entrada y salida planteadas en esta investigación, considerando que ofrecen oportunidades de mejoras para mejorar sus diferentes tipos de eficiencia. En este sentido sería importante que estas EPS establecieran alianzas para fortalecer la estructura de sus servicios y mejorar su eficiencia y competitividad como lo señalan otros estudios [13]. 
Estos resultados se constituyen en referentes y criterios para la toma de decisiones por parte de los directivos de las EPS en Colombia para que redireccionen sus decisiones referentes a las gestiones administrativas y técnicas que garanticen el incremento de las eficiencias de las cinco EPS que presentaron ineficiencias, por razones de toma de decisiones asociadas a las mezclas de los recursos utilizados, los retornos de escala y a problemas administrativos o puramente técnicos.

Asimismo esta investigación se constituye en un referente para la los usuarios de las EPS en Colombia, para que puedan analizar y evaluar la mejores EPS del régimen Contributivo y tomar decisiones referentes a la elección de la EPS más eficiente. En este sentido es importante señalar que la naturaleza en las instituciones de salud es compleja lo que requiere múltiples acciones y concepciones para mejorar la eficiencia de estas como lo proponen otras investigaciones [25]. Por otro lado, estudios en Portugal en el sector salud, destacan la necesidad de generar procesos de alianzas y sinergias entre las diferentes entidades de salud y la optimización de recursos disponibles, con el fin de mejorar la eficiencia de escala [13], lo que debe ser considerado por parte de las diferentes instituciones al buscar mejorar las diferentes eficiencias, de las entidades objeto de esta investigación.

Como proyección de esta investigación, se invita a los investigadores y directivos de las EPS a realizar un análisis causal entre las variables y dimensiones asociadas a la racionalidad y eficiencia técnica, administrativa y global. Y los rubros financieros que permitan priorizar sobre las variables que más inciden en la evaluación de la eficiencia global, que posibilite el mejoramiento en la utilización de los recursos por parte de las EPS del régimen Contributivo en Colombia, cuando se utilizan modelos de evaluación y medición de la eficiencia similares al utilizado en esta investigación con otras variables y rubros de entrada y salida.

Es importante señalar que para esta investigación se seleccionaron un grupo de variables de entrada y salida, con el fin de determinar los niveles de eficiencia del grupo de entidades objeto de estudio, lo que implica una limitación al momento de analizar la eficiencia del grupo de entidades. Por lo que se invita a los investigadores a analizar otros factores y variables que puedan afectar la eficiencia de estas organizaciones.

Como futuras líneas de investigación también, sería importante analizar otros factores asociados a estructuras de calidad y competitividad que otros investigadores han desarrollado [26-30] y estudiar como estas nuevas condiciones, inciden en el mejoramiento de la eficiencia. Además, analizar el rendimiento productivo o de eficiencia que las EPS del régimen Contributivo cuando se utilizan y analizan indicadores financieros para comprender el comportamiento de estas de un período a otro, cuando se utiliza la técnica de cálculo multivariado [30-33].

\section{REFERENCIAS}

[1] J.C. Soler, D.E. Martínez, M.J.L. Barceló and E. Loyola-Elizondo. "Information systems in health and health indicators: an integrating perspective". Med. Clin. (Barc). Vol. 134 $\mathrm{N}^{\circ}$ 1, pp. 3-9. Febrero 2010.

[2] M.C. Jaramillo and D. Chernichovsky. "Información para la calidad del sistema de salud en Colombia: una propuesta de revisión basada en el modelo israelî’. Estud. Gerenciales. Vol. $31 \mathrm{~N}^{\circ} 134$, pp. 30-40. Enero 2015.

[3] D. Chernichovsky and S.I. Prada."Ajustes a la arquitectura del sistema general de salud de Colombia: una propuesta". Estud. Gerenciales. Vol. $31 \mathrm{~N}^{\circ} 135$, pp. 163-170. Diciembre 2014.

[4] M. Reguanti. "Accesibilidad y eficiencia de la atención continuada en atención primaria de la salud". FMC - Form. Médica Contin. en Atención Primaria. Vol. $19 \mathrm{~N}^{\circ} 3$, pp. 115-116. Marzo 2012.

[5] M.L. Vázquez, E. Siqueira, I. Kruze, A. Da Silva and I.C. Leite. "Los procesos de reforma y la participación social en salud en América Latina”. Gac. Sanit. Vol. 16 No 1 , pp. 30-38. Enero 2002.

[6] I. Vargas, M. Vázquez, P. Molina, A. Mogollón and J. Unger. "Reforma, equidad y eficiencia de los sistemas de salud en Latinoamérica. Un análisis para orientar la cooperación española. Informe SESPAS 2008". Gac. Sanit. Vol. 22, pp. 223-229. Abril 2008.

[7] A. Suárez."Reflexiones acerca del uso de los conceptos de eficiencia, eficacia y efectividad 
en el sector salud". Rev. Cuba. Salud Publica. Vol. $26 \mathrm{~N}^{\circ}$ 1, pp. 50-56. Enero 2000.

[8] P. Cabello and Á. Vega. "Análisis de la eficiencia hospitalaria por Comunidad Autónoma en el ámbito del Sistema Nacional de Salud". Investig. Reg. N ${ }^{\circ} 28$, pp. 147-158. Enero 2014.

[9] J. Tarrés, A. Mainar, J. López, R. Navarro, J. Codes and J. González. "Estudio comparativo de la eficiencia, medida a partir de los Ambulatory Care Groups, entre 4 centros de salud". Atención Primaria. Vol. $38 \mathrm{~N}^{\circ} 5$, pp. 275-282. Septiembre 2006.

[10] T. Fontalvo. "Eficiencia de las entidades del regimén subsidiado en Colombia por medio de análisis envolvente de datos". Rev. Univ. y Salud. Vol. 16 N$^{\circ} 1$, pp. 35-46. Mayo 2014.

[11] F. López. "Efficiency-based healthcare. General concepts on economic evaluation of healthcare interventions". Enfermería Clínica. Vol. 19 No 1, pp. 35-42. Enero 2009.

[12] E. García, J. Solves and M. Mora. "Efficiency indicators to contribute to sustainability of health services in Spain". Rev. Calid. Asist. Vol. $29 \mathrm{~N}^{\circ}$ 5, pp. 287-90. Enero 2014.

[13] H. Azevedo and C. Mateus. "Economias de escala e de diversificação: uma análise da bibliografia no contexto das fusões hospitalares". Rev. Port. Saúde Pública. Vol. $32 \mathrm{~N}^{\circ}$ 1, pp. 106-117. Enero 2014.

[14] J. García, G. Rodriguez and A. García. "Eficiencia técnica de los centros avanzados de atención primaria de la salud de Tabasco (CAAPS). Aplicación del método de optimización análisis envolvente". Salud en Tabasco. Vol. $14 \mathrm{~N}^{\mathrm{o}}$ 3, pp. 782-791. 2008.

[15] R. José and C. Salinas. "Medición de la eficiencia y productividad de la red de Servicio de Salud, del SILAIS Carazo 2010-2011, mediante la metodología Análisis envolvnete de Datos (DEA)". Rev. Investig. en ciencias ecónomicas. UNAM Manag. Vol. 2 No 3, pp. 82-105. Abril 2014.

[16] J. Soares, L. Angulo, J. Da Silveira and E. Gomes. "About negative efficiencies in Cross Evaluation BCC input oriented models". Eur. J. Oper. Res. Vol. $229 \mathrm{~N}^{\circ}$ 3, pp. 732-737. Septiembre 2013.

[17] C.M. Martínez and I. Raja. "Medida de la eficiencia en entidades no lucrativas: un estudio empírico para fundaciones asistenciales". Rev. Contab. Vol. $17 \mathrm{~N}^{\circ} 1$, pp. 47-57. Enero 2014.

[18] V. Delimiro and F. Gómez. "Evaluacion de la eficiencia relativa en el uso de recursos de la universidades públicas colombiana mediante la metodología Data Envelopment Analysis". Universidad de los Andes. 2006.

[19] W.W. Cooper, L.M. Seiford and K. Tone. "Introduction to Data Envelopment Analysis and its uses". 1st ed. New York, USA. Marzo 2006.

[20] V. Coll and O. Blasco. "Evaluación de la eficiencia mediante análisis Envolvente de Datos: Introducción a los modelos básicos". 1st ed. Universidad de Valencia. Valencia, España. Septiembre 2006.

[21] D.A. Marín and L.Á. Campos. "Estructura organizacional y relaciones interorganizacionales: análisis en Instituciones Prestadoras de Servicios de Salud Públicas de Colombia”. Estud. Gerenciales. Vol. 31 No 134, pp. 88-99. Enero 2015.

[22] J.Ligarda. "La eficiencia de las organizaciones de salud a través del análisis envolvente de datos". Microrredes de la Dirección de Salud IV Lima Este 2003". An. la Fac. Med. Vol. $67 \mathrm{~N}^{\circ}$ 2, pp. 142-151. Abril 2006.

[23] T. Fontalvo. "Evaluación de la productividad de las entidiades prestadoras de salud EPS del régimen subsidiado en Colombia, por medio de análisis discriminante". Hacia la promoción en Salud. Vol. $17 \mathrm{~N}^{\circ}$ 2, pp. 60-78. Octubre 2012.

[24] C.A. Merlano and I. Gorbanev. "Health system in Colombia: a systematic review of literature Sistema". Rev. Gerenc. en Polit. Salud. Vol. $12 \mathrm{~N}^{\circ}$ 571, pp. 74-86. Enero 2013.

[25] S. Peiró, J.J. Artells and R. Meneu, "Identification and prioritization of interventions to improve efficiency in the National Health System". Gac. Sanit. Vol. $25 \mathrm{~N}^{\circ} 2$, pp. 95105. Enero 2011.

[26] T. Fontalvo. "La calidad en los servicios ISO 9000: 2000". Corporación para la Gestión del Conocimiento ASD 2000. 2000.

[27] T. Fontalvo. "Herramientas efectivas para el diseño e implantación de un sistema de gestión de la calidad ISO 9000: 2000”. Corporación para la Gestión del Conocimiento ASD 2000. 2000. 
[28] T. Fontalvo. "La gestión avanzada de la calidad: metodologías eficaces para el diseño, implementación y mejoramiento de un sistema de gestión de la calidad". Corporación para la Gestión del Conocimiento ASD 2000. 2000.

[29] H. Mercado, T. Fontalvo and E. De la Hoz. "Análisis comparativo entre las cadenas productivas del sector textil-confecciones de la provincia de Jiangsu-China y el departamento del Atlántico-Colombia”. Ingeniare. Revista chilena de ingeniería. Vol. $19 \mathrm{~N}^{\circ} 3$, pp. 429441. Diciembre 2011.

[30] T. Fontalvo, J. Morelos and E. De la Hoz. "Aplicación del análisis discriminante para evaluar el mejoramiento de los indicadores financieros en las empresas del sector extracción de petróleo crudo y gas natural en Colombia". Revista Soluciones de Postgrado EIA. Vol. 1 N $^{\circ}$ 7, pp. 1-16. Enero 2011.
[31] T. Fontalvo, E. De la Hoz and J. Morelos. "Aplicación de análisis discriminante para evaluar el mejoramiento de los indicadores financieros en las empresas del sector alimento de Barranquilla-Colombia”. Ingeniare. Revista chilena de ingeniería. Vol. $20 \mathrm{~N}^{\circ}$ 3, pp. 320-330. Diciembre 2012.

[32] T. Fontalvo, J. Vergara and E. De la Hoz. "Evaluación del impacto de los sistemas de gestión de la calidad en la liquidez y rentabilidad de las empresas de la Zona Industrial Vía 40". Pensamiento \& Gestión. Vol. $20 \mathrm{~N}^{\mathrm{o}}$ 3, pp. 165-189. Enero 2012.

[33] J. Morelos, T. Fontalvo and J Vergara. "Incidencia de la certificación ISO 9001 en los indicadores de productividad y utilidad financiera de empresas de la zona industrial de Mamonal en Cartagena". Estudios Gerenciales. Vol. 29 N $^{\circ}$ 126, pp. 99-109. Marzo 2013. 